\title{
End-to-End Performance Analysis using Engineering Confidence Models and a Ground Processor Prototype
}

\author{
Klaus-Werner Kruse, Maximilian Sauer, Thomas Jäger, Alexandra Herzog, Michael Schmitt, \\ Markus Huchler \\ Airbus DS GmbH, Friedrichshafen, Germany \\ Kotska Wallace, Michael Eisinger, Arnaud Heliere, Alain Lefebvre \\ European Space Agency, Noordwijk, The Netherlands \\ Mat Maher, Mark Chang, Tracy Phillips, Steve Knight \\ Surrey Satellite Technology Ltd., Guildford, UK \\ Bryan T.G. de Goeij, Frits van der Knaap, Adriaan Van’t Hof \\ TNO, Delft, The Netherlands
}

\begin{abstract}
The European Space Agency (ESA) and the Japan Aerospace Exploration Agency (JAXA) are co-operating to develop the EarthCARE satellite mission with the fundamental objective of improving the understanding of the processes involving clouds, aerosols and radiation in the Earth's atmosphere.

The EarthCARE Multispectral Imager (MSI) is relatively compact for a space borne imager. As a consequence, the immediate point-spread function (PSF) of the instrument will be mainly determined by the diffraction caused by the relatively small optical aperture. In order to still achieve a high contrast image, de-convolution processing is applied to remove the impact of diffraction on the PSF. A Lucy-Richardson algorithm has been chosen for this purpose.

This paper will describe the system setup and the necessary data pre-processing and post-processing steps applied in order to compare the end-to-end image quality with the L1b performance required by the science community.
\end{abstract}

\section{INTRODUCTION}

Airbus DS has been selected by ESA as the prime contractor for the space segment, and coordinates the development of the platform as well as three European instruments, the Atmospheric Lidar (ATLID), the BroadBand Radiometer (BBR) and the Multispectral Imager (MSI).

The EarthCARE mission will feature two active instruments, ATLID and CPR. In order to achieve high SNR ratios with these instruments, the $\mathrm{S} / \mathrm{C}$ will be operating at a low earth orbit with a $400 \mathrm{~km}$ altitude, thus covering a significant spatial angle with reasonable reception telescope diameters. At such an altitude the atmospheric drag strongly reduces the possible mission durations, thus the EarthCARE S/C needed to be designed as compact as possible.

The paper at hand discusses the performance of the MSI instrument. The MSI instrument design implementation consists of two separate cameras mounted on a common optical bench. Surrey Satellite Technology Ltd. (SSTL) has been selected as the instrument prime for the overall instrument, and develops the Thermal InfraRed (TIR) camera in-house.

Image and Signal Processing for Remote Sensing XXI, edited by Lorenzo Bruzzone, Proc. of SPIE Vol. 9643, $964305 \cdot$ C 2015 SPIE · CCC code: 0277-786X/15/\$18 · doi: 10.1117/12.2194301 
The second camera, providing Visible, Near-infrared and Shortwave-infrared channels (VNS), has been subcontracted to the Netherlands Organisation for Applied Scientific Research (TNO).

Driven by the EarthCARE mission requirement for a compact design, the MSI design covers an overall footprint of only about $65 \mathrm{~cm} \times 40 \mathrm{~cm}$. To provide a comparison, the high-resolution MSI on-board of the Sentinel-2 mission is designed on a $200 \mathrm{~cm} \times 135 \mathrm{~cm}$ footprint area. As a result of the spatial constraints, the EarthCARE MSI had to be designed with comparably small aperture sizes, i.e. just large enough to meet the missions radiometric and SNR requirements with the given sampling distance and orbit altitude. At the same time, internal optical parameters as focal length and the size of the detector elements is the result of a dedicated tradeoff. A smaller focal length and detector size will initially decrease size, but come at the price of a more complex optical design. Past a certain threshold this will lead to the need for more components and therefore starts to increase size.

The VNS camera contains four dedicated CMOS dectors and two apertures with diameters of $4.85 \mathrm{~mm}$ and $10.47 \mathrm{~mm}$ respectively. Images are mapped by $\mathrm{f}=22.2 \mathrm{~mm}$ optics onto the $25 \mu \mathrm{m}$ pixel pitch detectors.

The TIR camera (Thermal InfraRed) records three channels on a single $\mu$-Bolometer detector. It utilises a 30mm aperture, a set of imaging optics with an effective focal length of $31 \mathrm{~mm}$, the detector pixel pitch being $35 \mu \mathrm{m}$.

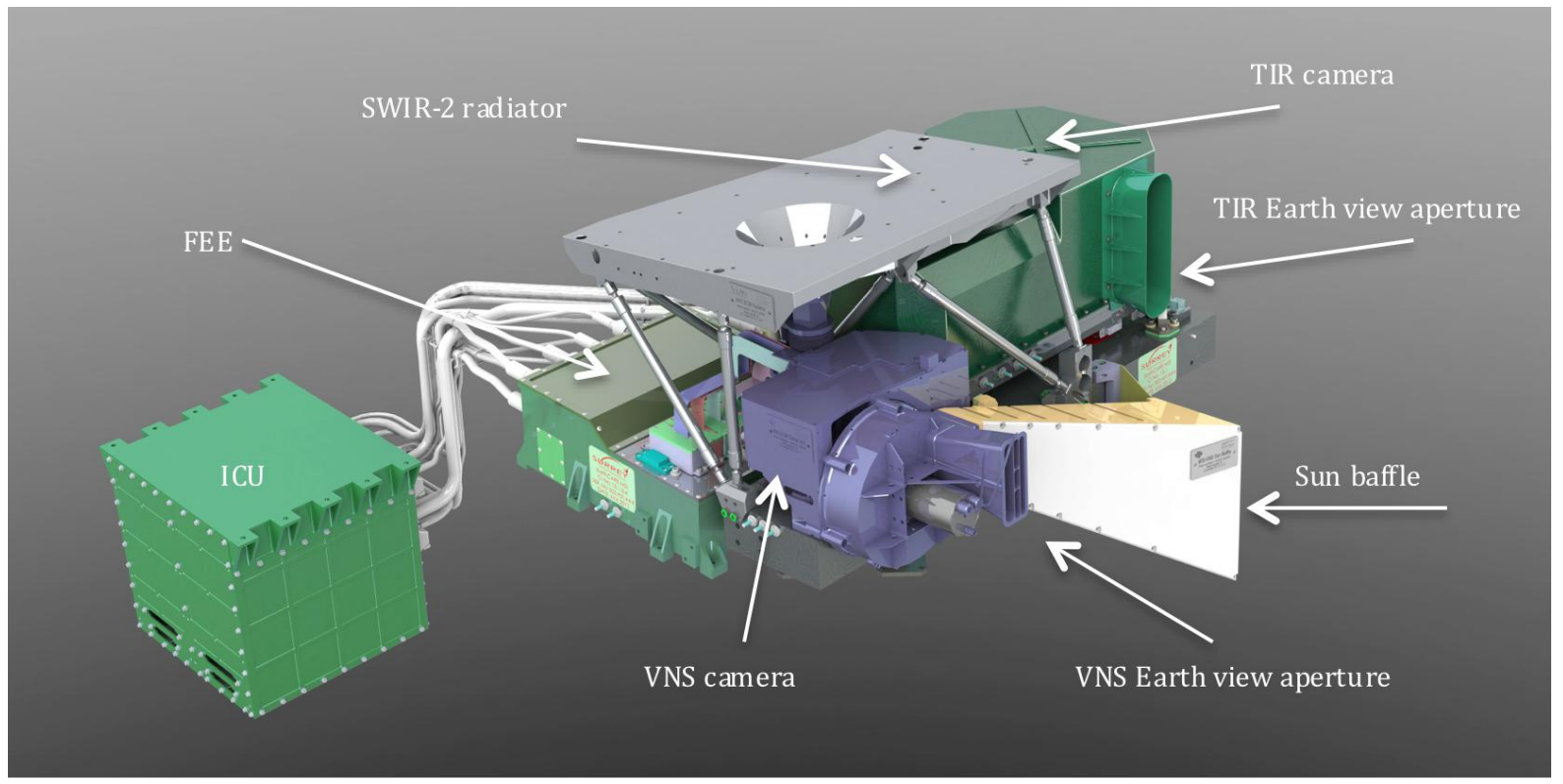

Figure 1 - 3D CAD model of the MSI

The amount of diffraction resulting from the small aperture diameters has a very noticeable impact on the instrument contrast and resolution of the EarthCARE MSI. The MSI instrument provides scene identification and classification as an input to a number of synergetic data products. As the active instruments have very well localised fields of view $(\sim 30 \mathrm{~m}$ for the Lidar and $<700 \mathrm{~m}$ for the Radar), MSI is specified to provide images with a high contrast in order to correlate well with the other instrument data. 
Contrast is defined via two requirements within the instrument specification.

Modulation Transfer Function:

The system modulation transfer function (MTF) shall be larger than 0.25 for all frequencies between $0 \mathrm{~km}^{-1}$ and Nyquist frequency at level $1 b$.

\section{Straylight}

The MSI requirements shall be achieved for spatial samples located at a distance larger than 2 SSD on each side of the step transition [from maximum radiance to low reference radiance].

In order to meet its requirements, the instrument needs to provide a measurement of the low reference radiance with a relative radiometric accuracy of $1 \%$ in the VNS channels, and of $0.25 \mathrm{~K}$ for the TIR channels. The stray light analysis of the instrument has quickly shown that the first challenge to meeting and verifying these requirements would not be the real stray light, but the amount of diffraction introduced by the size of the apertures. While the actual stray light and the detector cross-talk contributions to the low signal are acceptable to the error budget 2 SSD (Spatial Sampling Distances) from the edge, diffraction prevents the instrument design from compliance at level 0 for up to 14 SSD from the edge.

Table 1 below shows the relative effect of the three main contributors to the contrast for channel 9 (12 $\mu \mathrm{m}$ wavelength), as a function of the sample distance from the signal edge described above:

Table 1 - Temperature Error at the low reference signal side of a signal dropping edge - acceptable Contributions in green, excessive contributions in red

\begin{tabular}{|c|c|c|c|c|c|}
\hline SSD & Stray Light & $\begin{array}{l}\text { Aperture } \\
\text { Diffraction }\end{array}$ & $\begin{array}{l}\text { Detector } \\
\text { Cross-Talk }\end{array}$ & $\begin{array}{l}\text { Combined } \\
\text { "stray light" }\end{array}$ & $\begin{array}{c}\text { Temperature } \\
\text { Error }(\mathrm{K})\end{array}$ \\
\hline 1 & $0.01 \%$ & $1.57 \%$ & $1.00 \%$ & $2.58 \%$ & 6.86 \\
\hline 2 & $0.02 \%$ & $0.90 \%$ & $0.10 \%$ & $1.02 \%$ & 2.63 \\
\hline 3 & $0.02 \%$ & $0.63 \%$ & $0.01 \%$ & $0.66 \%$ & 1.65 \\
\hline 4 & $0.03 \%$ & $0.49 \%$ & $0.00 \%$ & $0.52 \%$ & 1.23 \\
\hline 5 & $0.03 \%$ & $0.40 \%$ & $0.00 \%$ & $0.43 \%$ & 0.97 \\
\hline 6 & $0.04 \%$ & $0.33 \%$ & $0.00 \%$ & $0.37 \%$ & 0.79 \\
\hline 7 & $0.04 \%$ & $0.29 \%$ & $0.00 \%$ & $0.33 \%$ & 0.66 \\
\hline 8 & $0.04 \%$ & $0.25 \%$ & $0.00 \%$ & $0.29 \%$ & 0.56 \\
\hline 9 & $0.04 \%$ & $0.22 \%$ & $0.00 \%$ & $0.26 \%$ & 0.49 \\
\hline 10 & $0.04 \%$ & $0.20 \%$ & $0.00 \%$ & $0.24 \%$ & 0.43 \\
\hline 12 & $0.04 \%$ & $0.16 \%$ & $0.00 \%$ & $0.20 \%$ & 0.34 \\
\hline 14 & $0.04 \%$ & $0.14 \%$ & $0.00 \%$ & $0.18 \%$ & 0.27 \\
\hline 16 & $0.03 \%$ & $0.12 \%$ & $0.00 \%$ & $0.15 \%$ & 0.22 \\
\hline 18 & $0.03 \%$ & $0.10 \%$ & $0.00 \%$ & $0.13 \%$ & 0.18 \\
\hline 20 & $0.03 \%$ & $0.08 \%$ & $0.00 \%$ & $0.11 \%$ & 0.14 \\
\hline 25 & $0.03 \%$ & $0.06 \%$ & $0.00 \%$ & $0.09 \%$ & 0.08 \\
\hline 30 & $0.02 \%$ & $0.04 \%$ & $0.00 \%$ & $0.06 \%$ & 0.03 \\
\hline 35 & $0.02 \%$ & $0.00 \%$ & $0.00 \%$ & $0.02 \%$ & -0.06 \\
\hline 40 & $0.02 \%$ & $0.00 \%$ & $0.00 \%$ & $0.02 \%$ & -0.06 \\
\hline 45 & $0.02 \%$ & $0.00 \%$ & $0.00 \%$ & $0.02 \%$ & $=0.05$ \\
\hline 50 & $0.02 \%$ & $0.00 \%$ & $0.00 \%$ & $0.02 \%$ & -0.04 \\
\hline
\end{tabular}


Reducing diffraction through hardware changes would have required to scale the TIR aperture up by a factor of 7 (14SSD / 2SSD). As it was clear that an instrument with a $210 \mathrm{~mm}$ aperture could not be compatible with the need for a compact instrument for the EarthCARE mission, deconvolution has been proposed as a processing step in order to achieve the specified contrast for level $1 \mathrm{~b}$ data.

Feasibility of the deconvolution via the Lucy-Richardson Algorithm has been assessed based on simulated data during the design phase, and has been found to be very promising. Nevertheless, the complexity of such an iterative, non-local algorithm is significant, and the processing results can be affected by a number of parameters, such as the image noise or the determination accuracy of the point-spread function.

Therefore, in order to increase confidence in the performance of the deconvolution algorithm specification and implementation, it has been decided to evaluate the L1 performance as soon as possible in the programme, by using real images recorded with an engineering confidence model (ECM) of the instrument in combination with an early version of the EarthCARE ground processor prototype (ECGP V1).

\section{THE ENGINEERING CONFIDENCE MODELS}

The purpose of the MSI Engineering Confidence Model is to de-risk the development of the instrument. The ECM is intended to validate the overall instrument concept prior to proceeding with the Proto-Flight Model. It consists of an ECM TIR camera (Figure 2) and an ECM VNS camera (Figure 3). It also incorporates the Optical Bench Module and an Elegant Breadboard (EBB) for the Front End Electronics. The VNS ECM camera does not include the NIR and SWIR-2 channels.

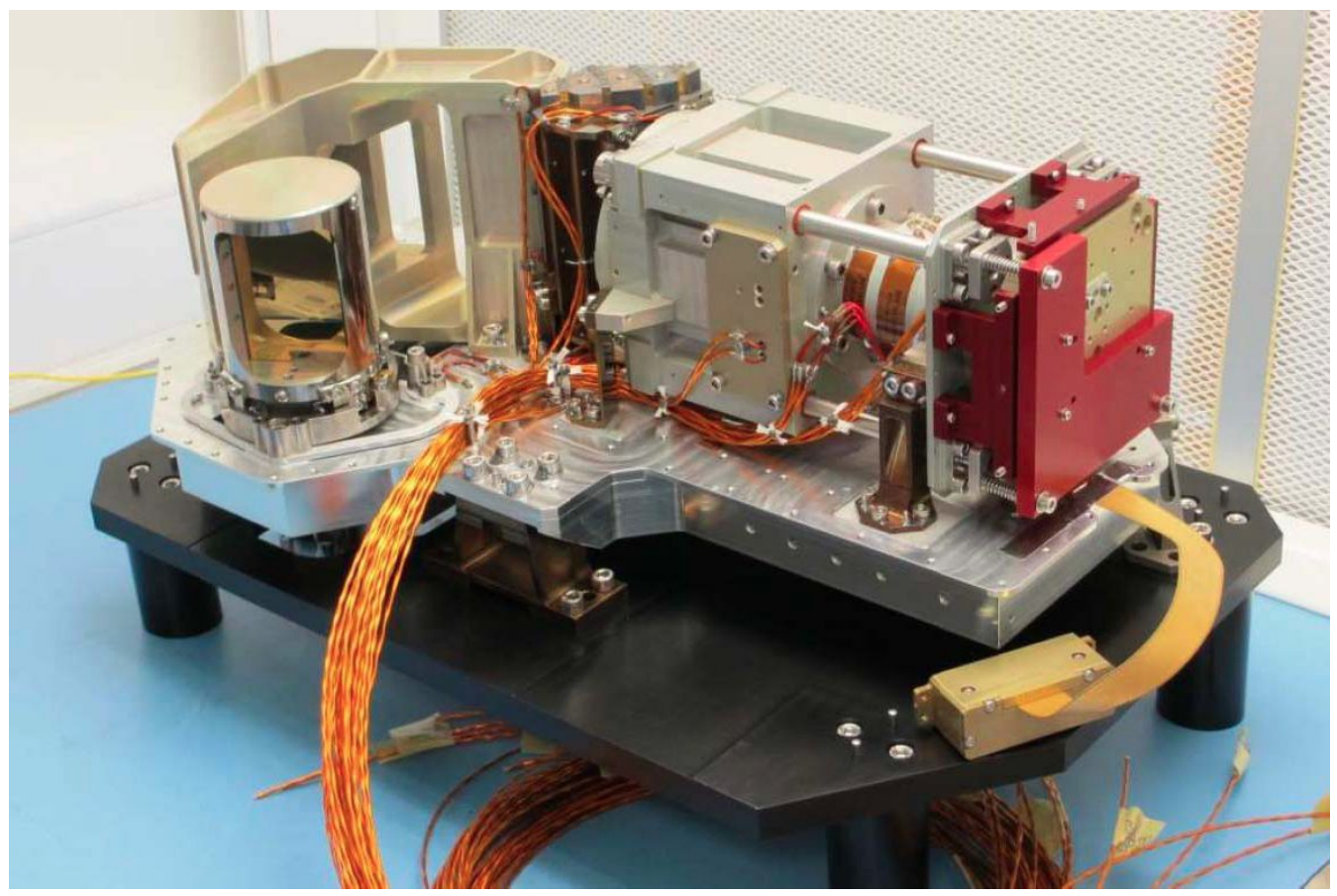

Figure 2 - TIR ECM camera 


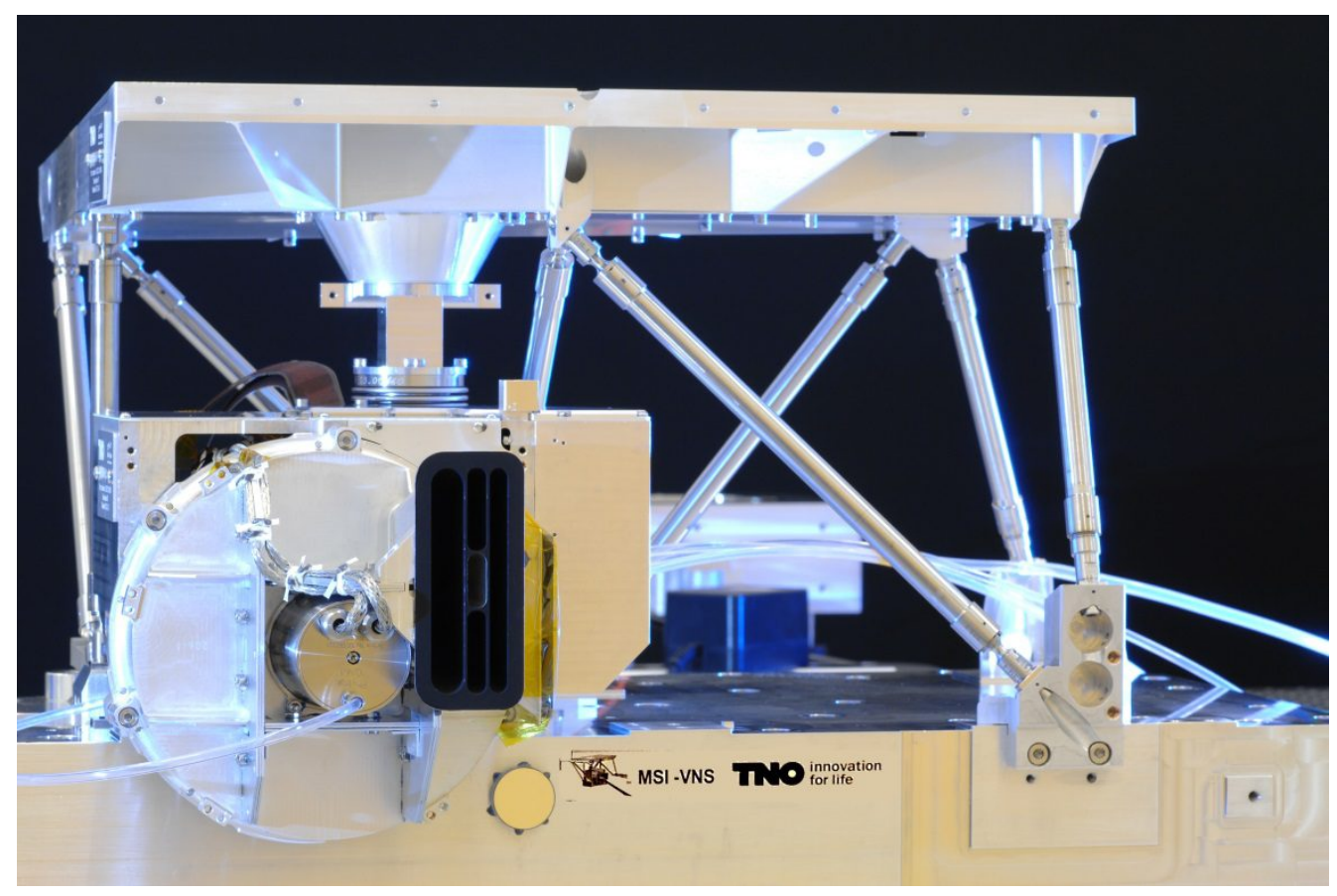

Figure 3 - VNS ECM camera. Sun baffle not assembled.

The electrical and electronic setup of the ECM differs from the in-flight configuration concerning the handling of digitised data, but are the same in regards of the analogue signal processing from the detectors up to the Front-End Electronics (FEE). In the flight setup, the FEE will be connected to an Instrument Control Unit (ICU), which will preprocess and packetize the science data together with auxiliary information such as time stamps and instrument mode information. The Instrument Source Packets (ISPs) will then be handed over to the satellite Mass Memory \& Formatting Unit (MMFU) for storage.

In the ECM setup used for performance tests (shown in Figure 4 for the VNS camera), the FEE is connected through a dedicated FEE interface unit to a Data Acquisition \& Processing Block (DAPB), basically a PC with the capability to visualise and post-process the instrument detector readouts on the go. The test data are then stored in image and/or spreadsheet formats. 


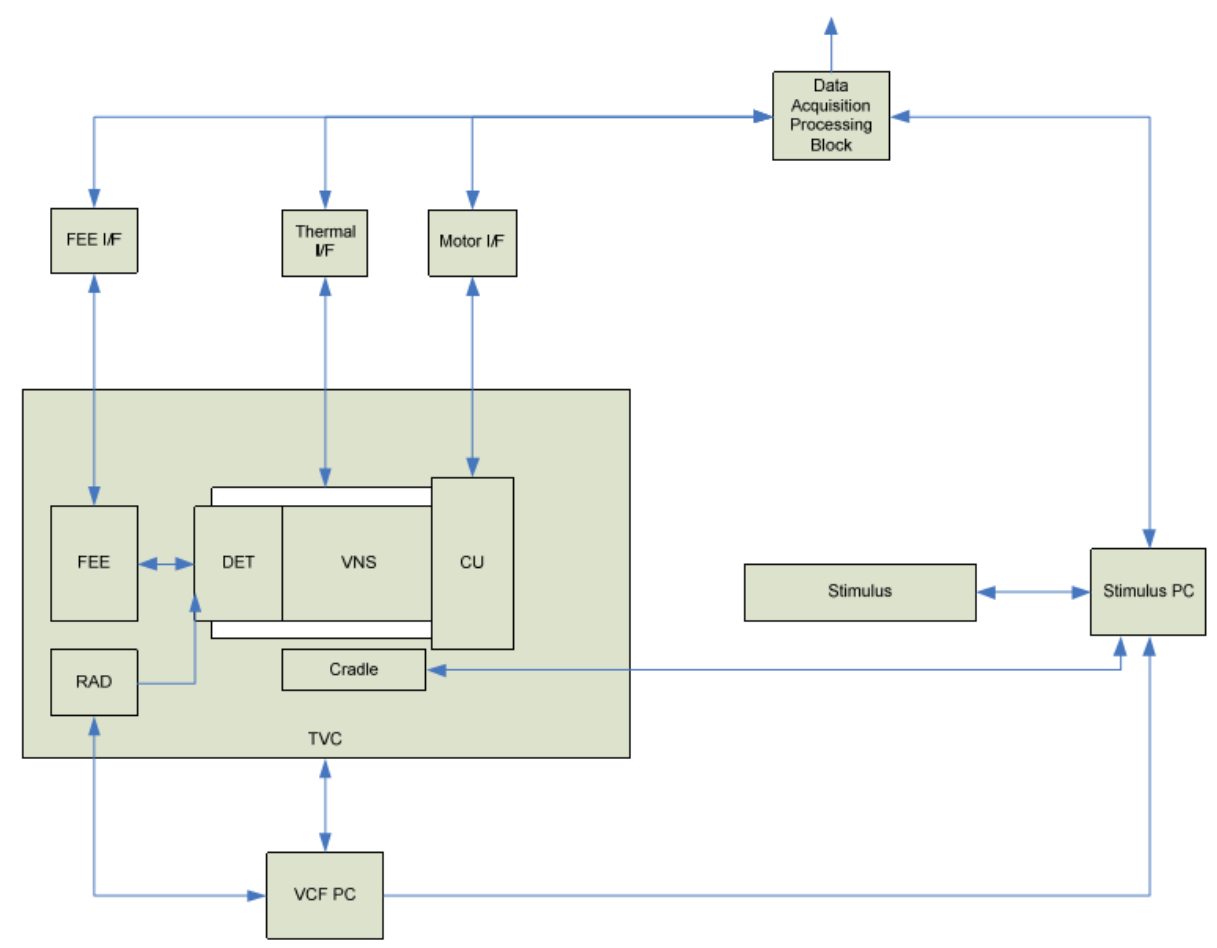

Figure 4 - VNS ECM performance testing setup

\section{THE GROUND PROCESSOR PROTOTYPE}

The purpose of the ECGP is to simulate the ground processors processing and generate L0/L1 products for all instruments in all instrument modes. The ECGP will also be able to detect/model errors in the Measurement Data Stream and to record these errors in the Products' PCDs.

The goals of the ECGP are inter alia:

1. to validate the L1 Processing Algorithms;

2. to demonstrate the L1 Products Quality:

a. on ground by processing the Measurement Data Stream generated by the ECM;

b. in orbit by processing the real ISPs.

The figure below shows the ECGP chain as well as the data flows to and from the ECGP. The ECGP DHP which constitutes the core processing functionality is broken down into its main components, namely:

- A Annotator and Error Injector (AEI) Logical Function that implements relevant effect of the down-link;

- The ECGP Front-End (FEP) that is in charge of generating L0 Products for all EarthCARE Instruments;

- The Core L1 Processors, each in charge generating the L1 Products for one EarthCARE Instrument. 


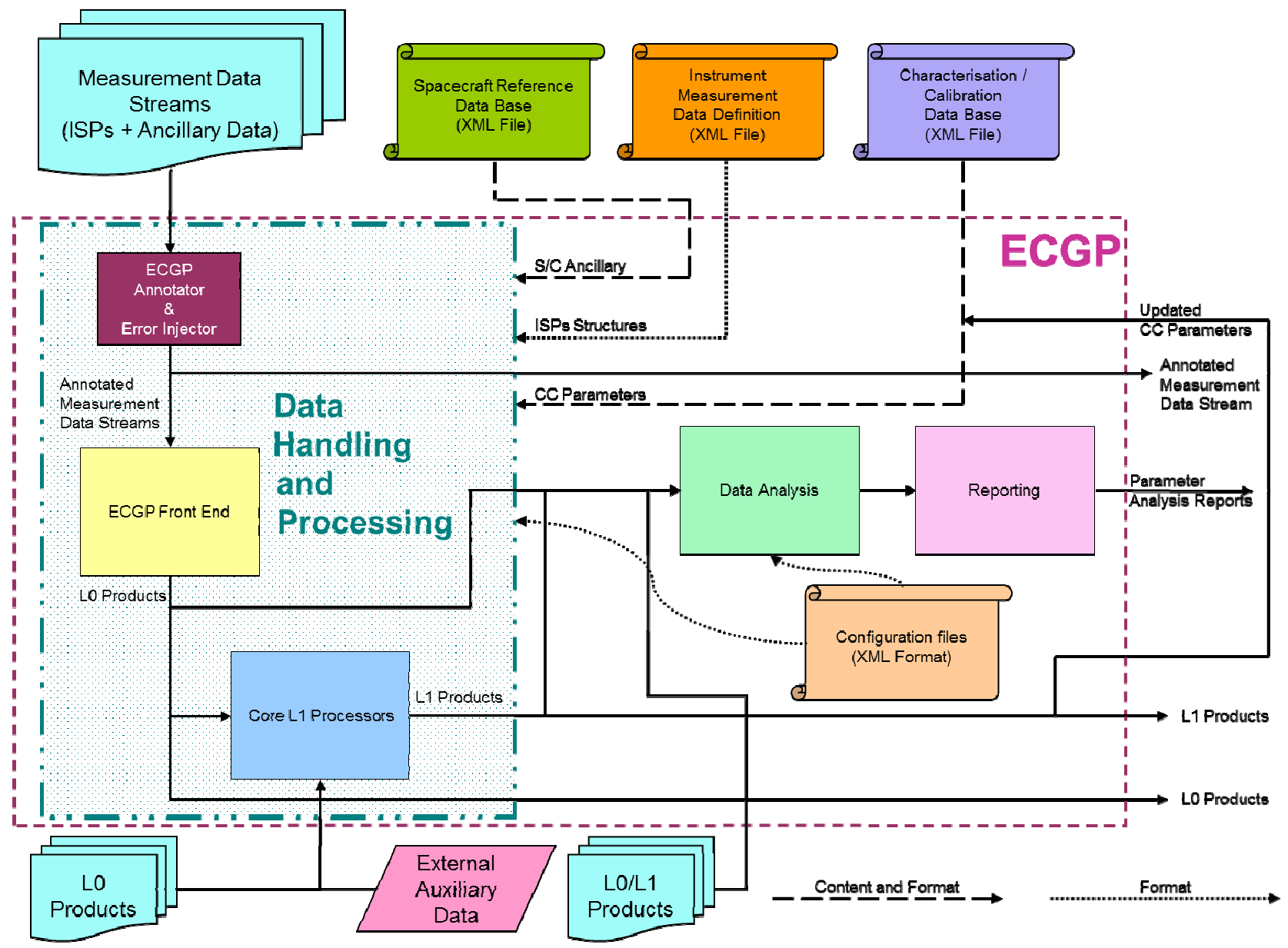

Figure 5 - ECGP Logical Decomposition and Data Flows

The ECGP AEI adds the Annotation Headers to the ISPs in the different Measurement Data Streams before the L0 processing starts. The AEI is also able to inject different kind of errors to the data stream in order to allow the verification of the ECGP error handling.

The ECGP Front End performs syntax and semantic checks on the input Measurement Data Stream: this will be checked for corrupt, missing, out-of-order etc. ISPs and Ancillary Data. The ECGP Front End will also generate L0 Products for all EarthCARE Instruments. The L0 Products will contain ISPs as well as Ancillary Data. Any corrupt, missing, duplicated or otherwise invalid ISPs or Ancillary Data will be either removed or marked as such.

The Core L1 Processors are in charge of ingesting the L0 Products from the ECGP Front End and of generating the L1 Products. There will be one dedicated Core L1 Processor for each EarthCARE Instrument.

L1 Products will contain inter alia:

- $\quad$ ATLID

○ Pure Attenuated Backscatter for the Mie co-polar, the Mie cross-polar and the Rayleigh Channels

- Range-corrected Attenuated Backscatter for the Mie co-polar, the Mie cross-polar and the Rayleigh Channels 
- $\mathrm{CPR}$

- apparent per unit volume radar cross-section

- Doppler Velocity of particles/droplets

- Doppler Velocity Spectral Width

- MSI

- Brightness Temperature (LW Channels)

- "Top Of Atmosphere" Radiance (SW Channels)

- BBR

- TOA Radiance (for both SW and TW Channels)

The MSI L1 Processor has been used in conjunction with the Annotator and Error Injector and the Front End Processor for the processing of the ECM data sets.

\section{ADDITIONAL PROCESSING STEPS AND TEST EXECUTION}

Due to the differences between the ECM and PFM configurations described in section 2, additional processing steps are required in order to create L0 products which can serve as an input to the ECGP. The detector readout data are available, but the additional information which is contained in an ISP, such as the data time stamp and instrument flags had not been generated during the test.

TNO and SSTL have thus manually generated the ISP headers with meaningful auxiliary data for the test data included. They also needed to re-sort the data, as the data for all 8 channels ( 7 science channels +1 TIR reference band) are interleaved within the ISP, with the data for all channels at a certain timestamp being provided before any data for the same channel at the next time step.

Radiometric accuracy requirements are specified to be met without consideration of noise. In order to judge the noisefree instrument performance, the images of the stray light tests have been averaged over significant periods of time, resulting in a single input scene for the processor with a greatly increased SNR.

In addition to the test scenes directly measured and provided for processing, the test results have also been used for the generation of a Characterisation and Calibration DataBase (CCDB). Most relevant for the article at hand, the CCDB contains the actual instrument Point-Spread-Function and the overall noise characteristics measured on the ECM models.

SSTL have generated one additional test scene using their instrument simulator. The MTF requirement for the MSI instrument is defined as the imaged contrast of a scene in which the signal intensity oscillates with a $\sin (x)$ function. As such a scene would be extremely difficult to implement as a physical input, it was instead generated within the simulator and folded with the real PSF measured on the ECM instrument.

Combined test data have been delivered in a batch for each test scenario, which consisted of three individual product files: a dark field measurement for offset correction, a white field measurement for gain correction, and the actual reference scene in text/spreadsheet format.

Pre-processing continued at Airbus DS with a MATLab based parser providing the required input-format for the ECGP. Here, individual products have been parsed into a single binary file for each test scene and checked for compliance with the definitions in the ICD. Resulting binary ISP time stamps have been shifted to the time stamp of already available orbit scenarios which had been provided by ESA for ECGP testing.

The modified ISPs, ancillary data and the instrument CCDBs have then been provided as inputs to the ECGP V1 for a processing cycle via the annotator error injector (AEI), front end processor (FEP), and finally the ECGP core processors. 


\section{Test scenes and performance evaluation}

Two relevant test scenes will be considered within this article in order to judge the impact of the deconvolution algorithm on the MSI instrument performance. The first scene provides the classical measure for the contrast of a camera, a contrast variation at the Nyquist frequency of the detector. As described above, this scene has been simulated to be an actual sinusoid variation of intensity.

In order to maximise the information content of the scene, the direction of the variation has been slightly tilted by $2^{\circ}$ against the across-track direction of the instrument. Due to this angle, the image contains the instrument response at a high number of different phasings between the scene maxima/minima and the detector sampling grid. As a side-effect of this tilt, the actual spatial frequency in the along-track direction is slightly over the Nyquist frequency $\left(\sim 1.02 \mathrm{f}_{\text {nyq }}\right)$. This is sufficient for aliasing effects to be clearly visible in the image:

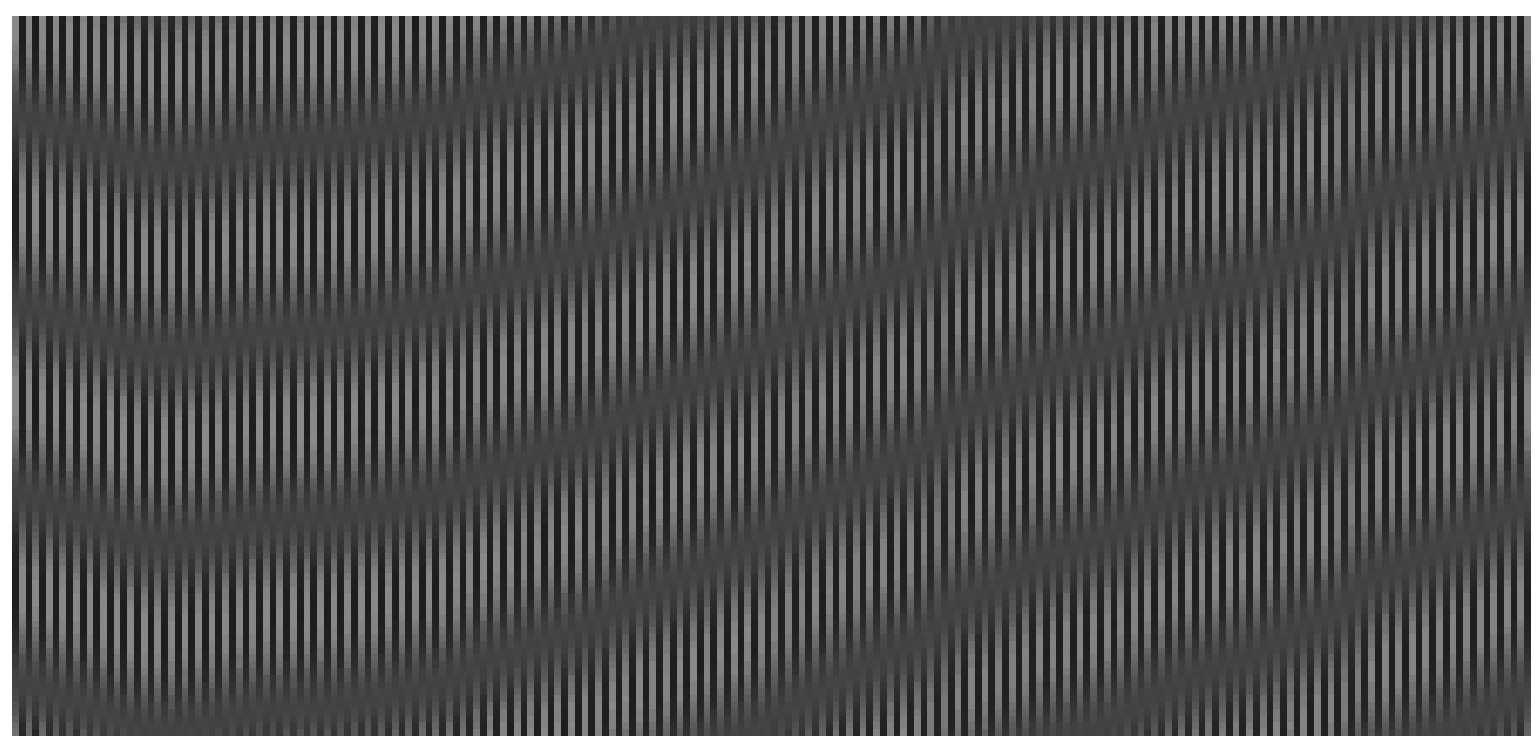

Figure 6 - MTF test scene, slightly tilted against the horizontal axis

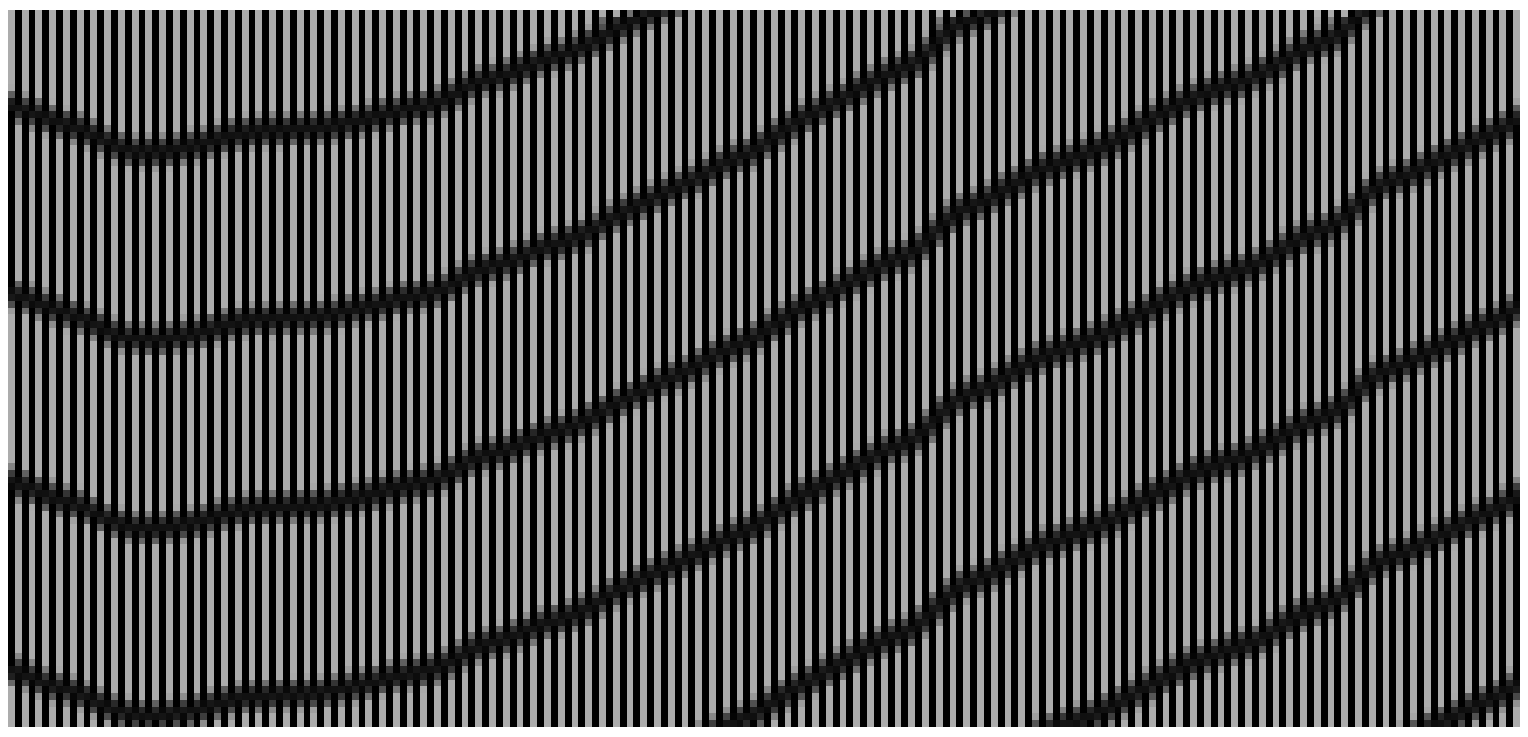

Figure 7 -Same Image as Figure 5, after deconvolution processing 
Figures 6 and 7 above show the MTF test scene before and after deconvolution processing respectively. The improvement to the overall contrast is clearly visible to the naked eye.

In order to generate a numerical assessment of the image MTF after deconvolution, the image data are reordered from the 2D images into 1D plots. To make full use of the image tilt and the resulting phasing information inherent in the data, all rows of the image are coordinate-shifted along the horizontal axis using the coordinate transformation $\xi=\mathrm{x}-$ $\sin \left(2^{\circ}\right)^{*} \mathrm{y}$. While in the image representation, values of $\mathrm{x}$ are always integer and represent the detector column number of the sample, the modified $\xi$ values are mainly non-integers, and the scene contrast variation is now perfectly perpendicular to the new $\xi$-axis. As such, the y-axis can be disregarded and a 1D-plot of the image intensity $S(\xi)$ represents an oversampling of the instrument response along any one detector row, shown in Figure 8 below:

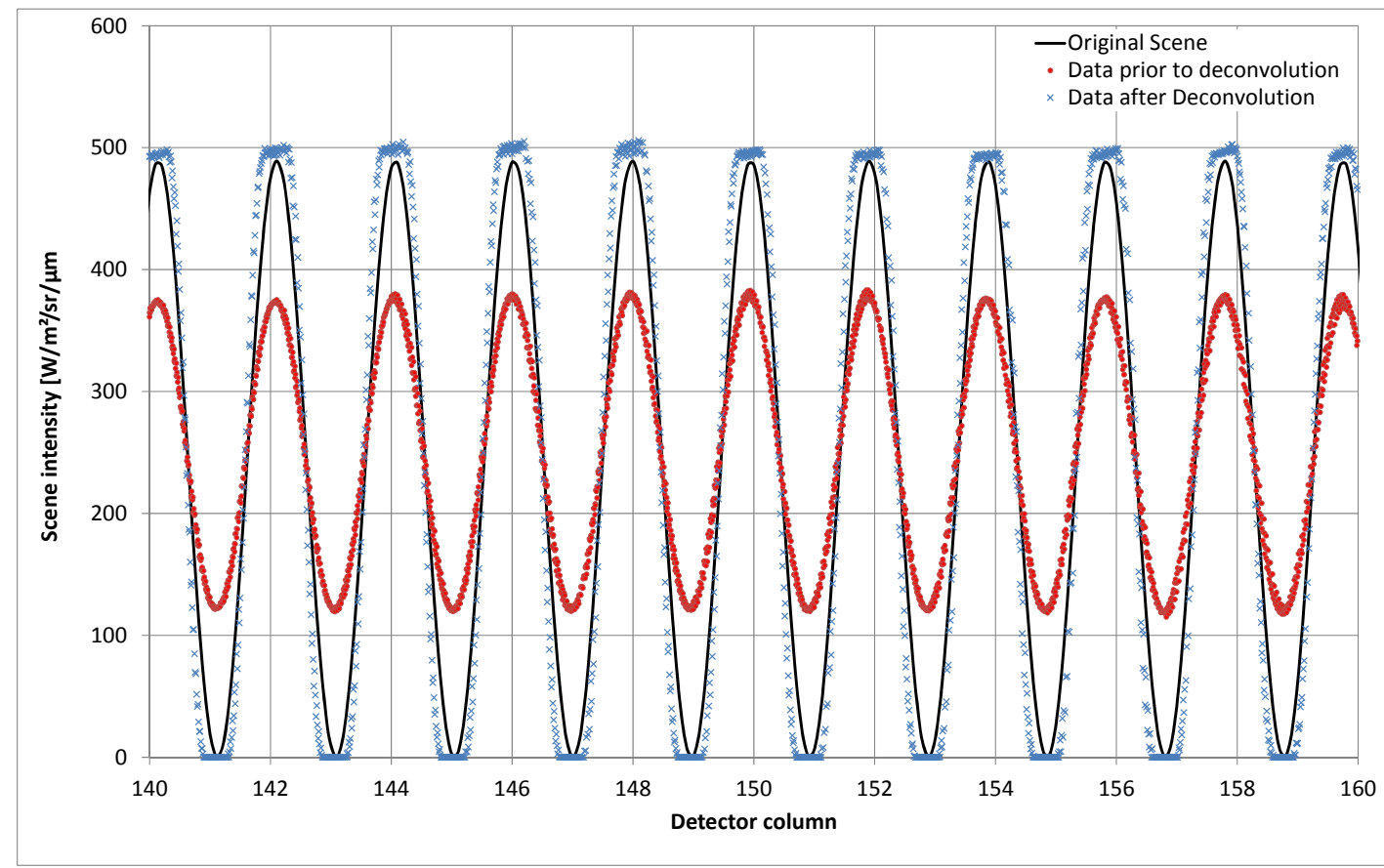

Figure 8 -1D representation of the image data in Figure 6

The figure provides a comparison of three sets of data.

1. A solid black line is plotted to represent the original intensity variation provided as scene definition to the instrument simulator.

2. Shown with red dots is the result of the simulation and initial processing. The data are modified by the measured ECM instrument MTF, and have been calibrated by the processor, but not de-convolved (data as shown in Figure 5). The contrast of the intensity variation is reduced to a factor of 0.52 .

3. Shown with small blue crosses is the final processing result as shown in Figure 6. After deconvolution, the contrast is actually higher than in the original scene, by a factor of 1.02 .

The overall contrast improvement, in direct response to the instrument requirement of "MTF @ Nyquist" is very significant: The processed signal is a quiet accurate representation of the original scene; even though the apparent oversampling within the 1D representation will of course not occur in reality, where the scene variation in y-direction contains real, independent information. 
One artefact is clearly visible within the test results; this is the formation of plateaus at the positions of minimum and maximum intensity. The root cause of this is the fact that negative values were generated as an (intermediate) result of the deconvolution for very low intensities within the original scene. As the Lucy-Richardson Algorithm is not robust against negative values, the current implementation of the deconvolution has set such results to exactly 0.0 . Through the iterative processing of the LR deconvolution, this ends up being mirrored by high intensity plateaus in the detector-rows which are affected by the truncation. An adaption of the algorithm implementation will be required in order to prevent this effect.

The second is a test scene directly measured during the VNS ECM tests. By illuminating a metal mask, a scene with strong contrast from $100 \%$ to $0 \%$ transmission is imaged by the camera. Again, moving the edge along the detector creates an angled contrast in the image providing an oversampling of the instrument response. In order to work around the algorithm restriction with values close to zero discussed above, we have added a small offset to this scene prior to deconvolution and subtracted it once more after deconvolution. Figure 9 and 10 show the recorded and averaged result of this measurement before and after deconvolution respectively.

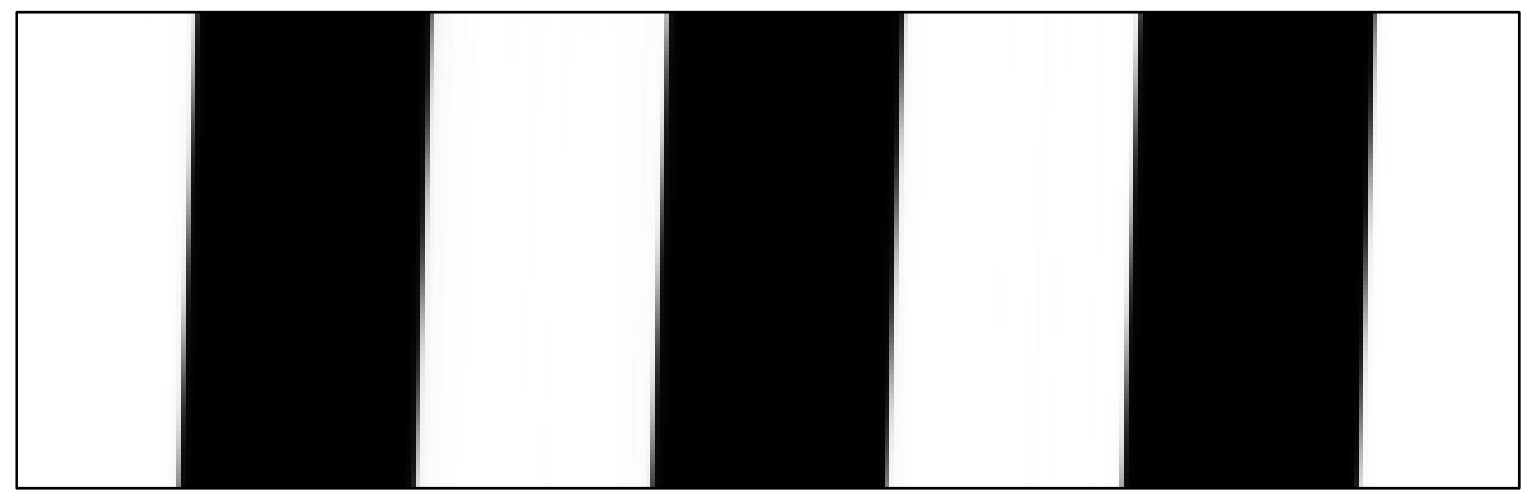

Figure 9 - Stray-light test scene

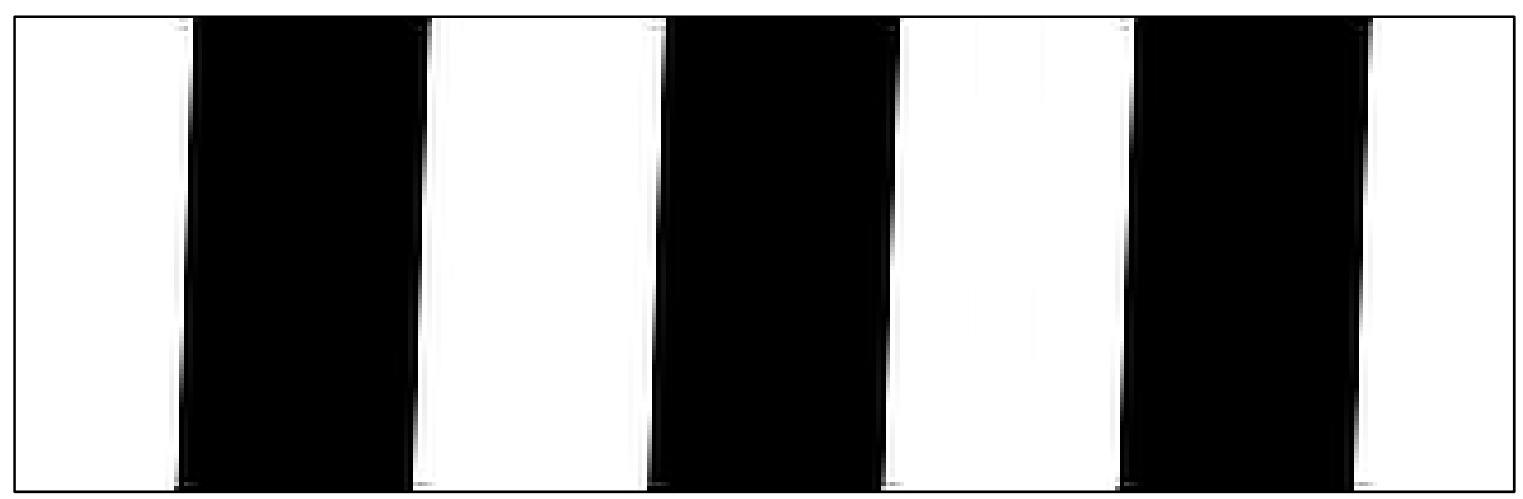

Figure 10 - Same as figure 9, after deconvolution processing

Differences due to the deconvolution are barely visible to the naked eye in the 2D image representation above, so once more we transformed the information into an 1D representation. 


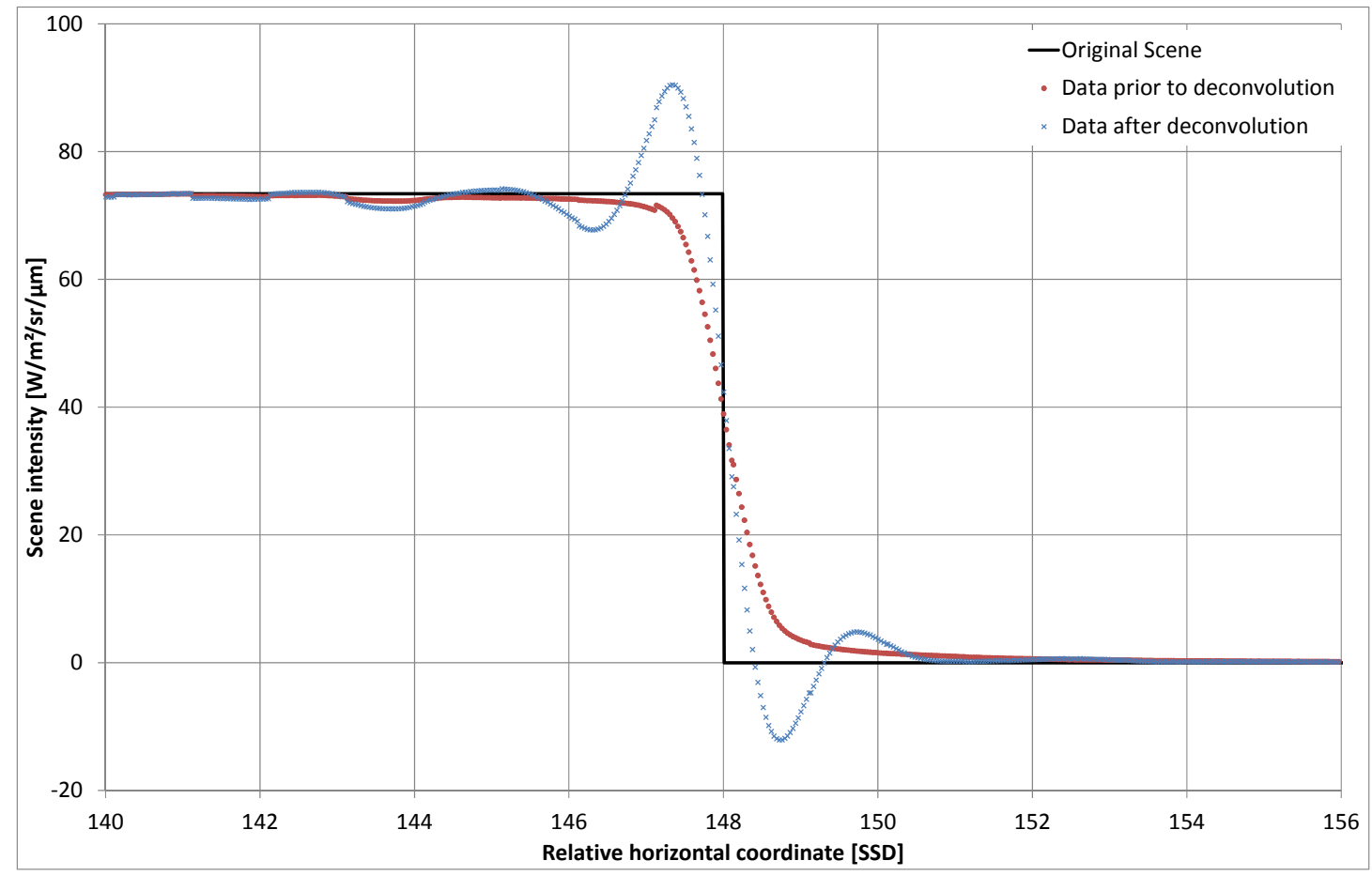

Figure 11 -1D Representation of the image data in Figure 9

The data plot in Figure 11 shows that deconvolution does increase the intensity slope at the scene edge, but it also introduces signal oscillations to both sides of the signal. This result was not predicted by the original numerical analysis conducted by SSTL during the instrument design phase. The original analysis process was:

1. Determine the worst-case system PSF, i.e. the one leading to the lowest MTF @ Nyquist.

2. Fold the specified straylight reference scene with the PSF

3. Add white noise corresponding to the worst-case SNR performance expected for the instrument

4. Apply Lucy-Richardson deconvolution of the data, using the exact same PSF as in step 1.

The results of this analysis are shown in Figure 12 below:
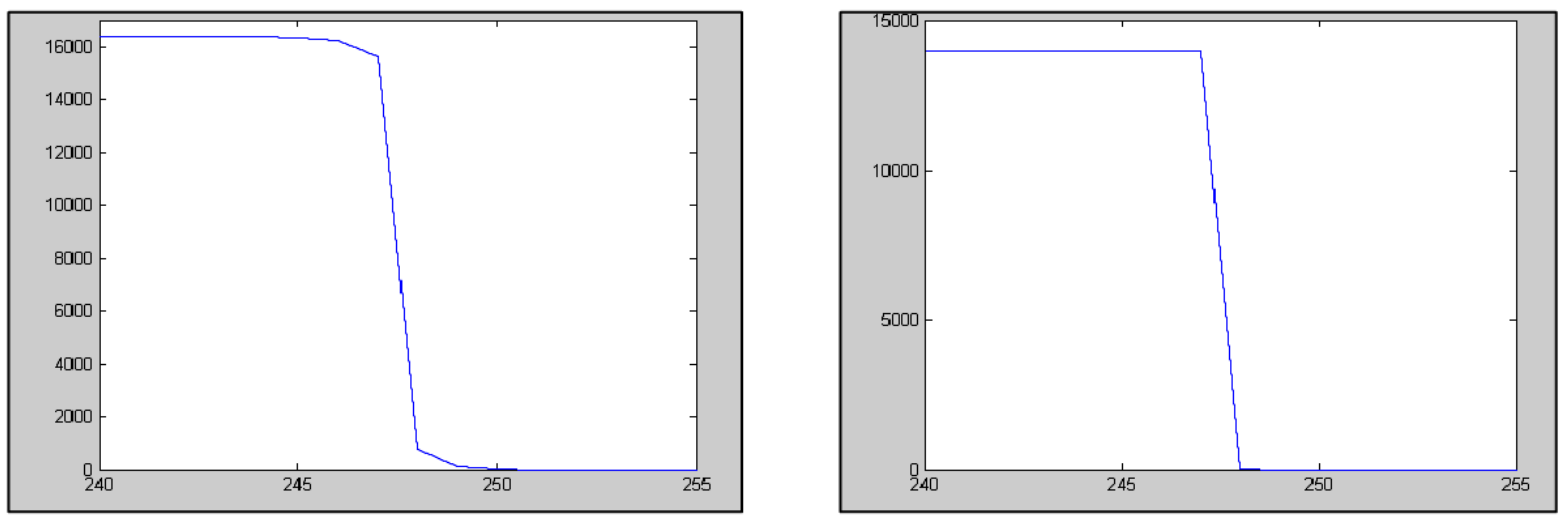

Figure 12 -Numerical Analysis of the MSI performance before (left) and after (right) deconvolution 
Under closer investigation it becomes clear that the difference in the end-to-end analysis and the initial numerical analysis is not the result of the specific instrument performance and algorithm implementation; but rather a result of using a less idealised scene in the testing than was defined in the specification and the early analysis. Tilting the high contrast scene by $2^{\circ}$ has introduced a wide range of phasing between the edge in the scene and the instrument sampling grid.

The results follow rather directly from the Nyquist-Shannon sampling theorem and the maximum likelihood approach of a Lucy-Richardson deconvolution. In short, sampling can produce the same image as a result of different scenes, if spatial variations with frequencies beyond the Nyquist frequency are involved. From a given image, the LR deconvolution will then attempt to reconstruct the most likely scene, that being the one which contains no spatial frequencies above f_nyq.

Thus, beyond the comparison shown in Figure 11 between the instrument data and the known original scene, it is also informative to compare the results to a low-pass filtered version of the scene, in which spatial frequencies beyond f_nyq are removed mathematically. This is done in Figure 13 below.

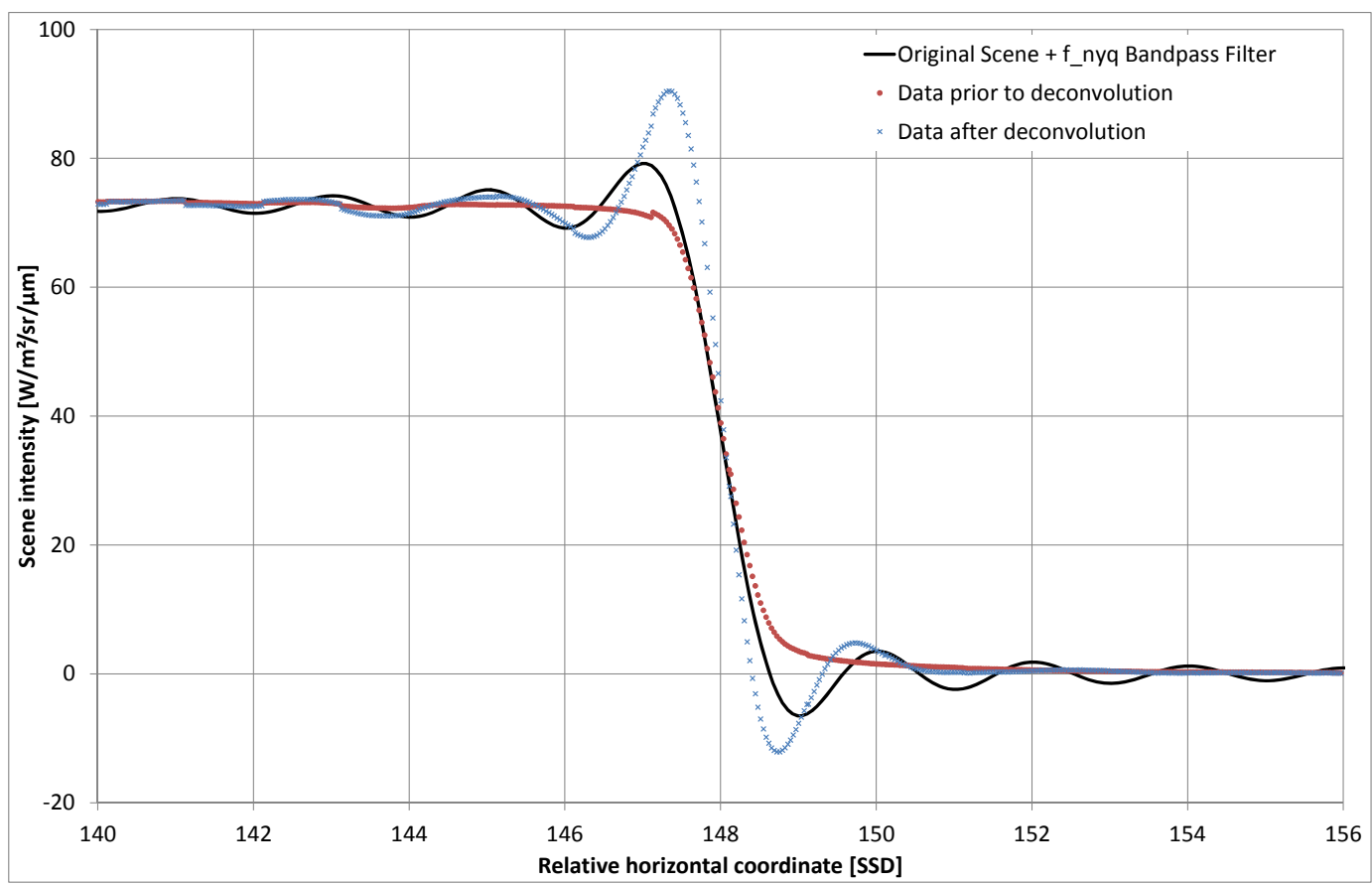

Figure 13 -MSI performances compared to an aliasing-free scene 


\section{CONCLUSION \#1: EarthCARE MSI L1 GEOMETRICAL PERFORMANCE}

Utilising Engineering Confidence Models of the MSI cameras and a Ground Processor Prototype implementation, the industrial consortium has been able to provide a detailed analysis of the end-to-end performance which will be provided by the instrument data once processed into L1products. Comparing the observed performance with the main contrast requirements of the instrument, two conclusions can be drawn.

Firstly, when the instrument images a well-behaved scene which only contains spatial frequencies below (or close to) the instrument Nyquist frequency, the L1 product will contain an excellent representation of the input scene. Indeed, the endto-end contrast achieved on the sample MTF scene is close to perfect, and goes beyond anything that could be achieved by any hardware implementation (with the same Nyquist frequency) without a dedicated contrast processing step.

Secondly, when imaging a scene that does contain a significant amount of spatial frequency beyond the Nyquist frequency (and even beyond the sampling frequency), matters are more complex. It is possible, even likely, that the endto-end implementation can show a formal compliance to the stray light requirement of imaging a high contrast scene with an edge perfectly aligned to one of the detector rows. At the same time the detailed analysis based on a slightly tilted version of the same scene has shown that the significance of the formal combination (a clear, simple requirement, and an exact verification) can be quite restricted.

\section{CONCLUSION \#2: Benefits of End-to-End Performance Analysis}

Using real measurement data generated from an Engineering Confidence Model of the MSI instrument under development, and applying the real processing through a prototype implementation of the Payload Data Ground Processor, we have achieved a greater insight into the real performance of the end-to-end chain than was previously provided by simulation and analysis alone.

This information is now available at a stage of the project where the hardware itself is designed and manufactured, but the calibration campaign of the instrument has not been concluded and a significant amount of time remains before the launch of the satellite.

The additional information gained from the analysis campaign can now be discussed with the customer, and further improvements on the processing of calibration data and on the definition of ground processing algorithms can be agreed well in advance of the in-orbit commissioning phase.

Additionally, we feel that using the instrument ECM models and the first implementation of the ECGP has achieved more for us than just generating a number to be compared to a requirement threshold: It has reminded us that reality is not constrained by our assumptions and simplifications, and that investigation of very specific examples may lead to a rather incomplete view of the imaging capabilities of the instrument design under consideration. 